\title{
Clinical Implication of Transaortic Mitral Pannus Removal During Repeat Cardiac Surgery for Patients With Mechanical Mitral Valve
}

\author{
Byungjoon Park, MD; Kiick Sung, MD, PhD; Pyo Won Park, MD, PhD
}

Background: This study aimed to evaluate the safety and feasibility of transaortic mitral pannus removal (TMPR).

\begin{abstract}
Methods and Results: Between 2004 and 2016, 34 patients (median age, 57 years; 30 women) with rheumatic disease underwent pannus removal on the ventricular side of a mechanical mitral valve through the aortic valve during reoperation. The median time interval from the previous surgery was 14 years. TMPR was performed after removal of the mechanical aortic valve $(n=21)$ or diseased native aortic valve $(n=11)$. TMPR was performed in 2 patients through a normal aortic valve. The mitral transprosthetic mean pressure gradient (TMPG) was $\geq 5 \mathrm{mmHg}$ in 11 patients, including 3 with prosthetic valve malfunction. Prophylactic TMPR was performed in 23 patients. There were no early deaths. Concomitant operations included 22 tricuspid valve surgeries (13 replacements, 15 repairs) and 32 aortic valve replacements ( 24 repeats, 8 primary). The mean gradient in patients who had mitral TMPG $\geq 5 \mathrm{mmHg}$ was significantly decreased from $6.46 \pm 1.1$ to $4.37 \pm 1.17 \mathrm{mmHg}$ at discharge $(P<0.001)$. No mechanical valve malfunction was apparent on last echocardiography.
\end{abstract}

Conclusions: TMPR is a safe and effective procedure for patients with malfunction or stenosis of a mechanical mitral valve and may be considered an alternative approach in patients with pannus overgrowth in such valves.

Key Words: Mitral valve; Prosthesis; Rheumatic heart disease; Surgery

$\mathbf{M}$ echanical prosthetic valves are still most commonly used in young patients with multiplevalve disease with a rheumatic etiology. Pannus formation in a mechanical valve is not common, but serious complications can result in valve malfunction and require cardiac reoperation during long-term follow-up. Fatal mechanical valve malfunction caused by pannus ingrowth has been extremely rare since the introduction of bileaflet mechanical valves.

Previously, our center reported that simultaneous aortic valve replacement (AVR) and mitral valve replacement (MVR) (i.e., double valve replacement [DVR]) with mechanical valves was associated with a higher aortic transprosthetic mean pressure gradient (TMPG) because of subaortic pannus formation than with isolated AVR. ${ }^{1-3}$ In those studies, increased aortic TMPG was associated with a late, significant tricuspid regurgitation (TR). Therefore, we aggressively corrected cases with a moderately stenotic aortic prosthesis using a new mechanical valve to prevent late TR and improve right heart failure at the time of tricuspid surgery. ${ }^{4,5}$ Pannus growth on the ventricular side of the prosthetic MV was frequently observed in patients with previous DVR at the time of repeat AVR. Several years prior, we also reported a case of successful transaortic removal of thickened anterior chordae, papillary muscle, and mitral pannus without repeat MVR in a patient with malfunction of a bileaflet mechanical MV. ${ }^{6}$

In the present study, the operative technique of transaortic mitral pannus removal (TMPR) was used in patients with a malfunction or stenosis of a mechanical MV and as a prophylactic procedure in patients with nonstenotic pannus protruding into the inlet orifice of the mechanical valve at the time of AVR. Recently, the American College of Cardiology/American Heart Association (ACC/AHA) guidelines define severe mitral stenosis as mean transmitral pressure gradient $\geq 5-10 \mathrm{mmHg} .^{7}$ In this study, the prophylactic procedure of TMPR was performed in patients with mitral TMPG $<5 \mathrm{mmHg}$.

The aims of this study were to evaluate the safety and feasibility of TMPR and to determine its clinical implications.

\section{Methods}

Patients

Between February 2004 and December 2016, 34 consecu-

Received May 2, 2017; revised manuscript received July 22, 2017; accepted July 27, 2017; released online August 31, 2017 Time for primary review: 51 days

Department of Thoracic and Cardiovascular Surgery, Samsung Medical Center, Sungkyunkwan University School of Medicine, Seoul, Korea

Mailing address: Pyo Won Park, MD, PhD, Department of Thoracic and Cardiovascular Surgery, Samsung Medical Center, Sungkyunkwan University School of Medicine, 81 Irwon-ro, Gangnam-gu, Seoul 06351, Korea. E-mail: pwpark@skku.edu

ISSN-1346-9843 All rights are reserved to the Japanese Circulation Society. For permissions, please e-mail: cj@j-circ.or.jp 


\begin{tabular}{|c|c|}
\hline & Mean \pm SD or $n(\%)$ \\
\hline \multicolumn{2}{|l|}{ Baseline characteristics } \\
\hline Age, years* & $57(50-63)$ \\
\hline Sex, female & $30(88)$ \\
\hline Body surface area, $\mathrm{m}^{2}$ & $1.54 \pm 0.13$ \\
\hline NYHA class III or IV & $12(35)$ \\
\hline Emergency operation & $2(3)$ \\
\hline Atrial fibrillation & $28(82)$ \\
\hline Hypertension & $3(9)$ \\
\hline Diabetes mellitus & $2(6)$ \\
\hline History of stroke & $10(29)$ \\
\hline Coronary artery disease & $1(3)$ \\
\hline \multicolumn{2}{|l|}{ No. of previous surgeries } \\
\hline 1 & $31(91)$ \\
\hline 2 & $3(9)$ \\
\hline \multicolumn{2}{|l|}{ Status of implanted mechanical valve } \\
\hline Double valve replacement & $24(71)$ \\
\hline Mitral valve replacement & $10(29)$ \\
\hline Interval from previous operation, years* & $14(11-17.3)$ \\
\hline \multicolumn{2}{|l|}{ Hospital of previous operation } \\
\hline Samsung Medical Center & $11(32)$ \\
\hline Other hospitals & $23(67)$ \\
\hline \multicolumn{2}{|l|}{$\begin{array}{l}\text { Preoperative laboratory and } \\
\text { echocardiographic data }\end{array}$} \\
\hline Hemoglobin, g/dL & $11.2 \pm 1.67$ \\
\hline Bilirubin, $\mathrm{mg} / \mathrm{dL}$ & $1.17 \pm 1.78$ \\
\hline Creatinine, $\mathrm{mg} / \mathrm{dL}$ & $0.92 \pm 0.32$ \\
\hline LVEF, \% & $61.2 \pm 8.97$ \\
\hline \multicolumn{2}{|l|}{ Mean transmitral pressure gradient, $\mathrm{n}(\%)$} \\
\hline$\geq 5 \mathrm{mmHg}$ & $11(32)$ \\
\hline$<5 \mathrm{mmHg}$ & $23(67)$ \\
\hline
\end{tabular}

*Median and interquartile range (25-75th percentile). LVEF, left ventricular ejection fraction; NYHA, New York Heart Association.

tive patients underwent pannus removal on the ventricular side of the MV through the AV; 4 patients who underwent only transseptal mitral pannus removal on the atrial side of the mitral mechanical valve were excluded. The median age of the patients was 57 years (interquartile range [IQR], $50-63$ years). Of these patients $30(88 \%)$ were women. A total of 28 patients $(82 \%)$ had associated atrial fibrillation. The underlying valve pathology was rheumatic heart disease in all patients. The status of the implanted mechanical valve replacement was DVR in 24 patients and MVR in 10 patients. The median time interval from the previous surgery was 14 years (IQR, 11-17.3 years). The detailed patient characteristics are described in Table 1 . This study was approved by the institutional review board, and the informed consent requirement was waived (IRB File no. 2017-02-132).

\section{Operative Procedure}

All operations were performed through a standard median sternotomy. Cardiopulmonary bypass was performed using bicaval cannulation under mild to moderate hypothermia. Repeat AVR was performed when the aortic TMPG exceeded $40 \mathrm{mmHg}$. Recently, aortotomy has become a routine procedure for the identification of significant sub-

\begin{tabular}{|lc|}
\hline \multicolumn{2}{|l|}{ Table 2. Operative Data for Transaortic Mitral Pannus } \\
Removal \\
Main indications for reoperation, $\mathbf{n}(\%)$ \\
Severe TR \\
Severe TR and AV pannus & $11(32)$ \\
AV pannus & $9(26)$ \\
Native AV disease & $6(18)$ \\
MV malfunction & $6(18)$ \\
Paravalvular MV leakage & $1(3)$ \\
Transaortic approach, $\mathbf{n}(\%)$ & $1(3)$ \\
After removal of prosthesis & \\
After removal of native AV & $24(71)$ \\
Normal functioning AV & $8(24)$ \\
MV malfunction, $\mathbf{n}$ (\%) & $2(6)$ \\
Complete immobilization of one cusp & \\
Delayed closure & $1(3)$ \\
Eccentric mitral regurgitation & $1(3)$ \\
MV pannus removal, $\mathbf{n}$ (\%) & $1(3)$ \\
Ventricular side & \\
Ventricular and atrial side & $26(76)$ \\
Concomitant operation, $\mathbf{n}(\%)$ & $8(24)$ \\
Repeat AVR & \\
AVR & $24(71)$ \\
TVR & $8(24)$ \\
TV repair & $13(38)$ \\
Maze operation & $15(44)$ \\
MV paravalvular leakage closure & $3(9)$ \\
Portic cross-clamp time, min & $1(3)$ \\
AV, aortic valve; AVR, aortic valve replacem & $184 \pm 47$ \\
\hline
\end{tabular}

AV, aortic valve; AVR, aortic valve replacement; MV, mitral valve; TR, tricuspid regurgitation; TV, tricuspid valve; TVR, tricuspid valve replacement.

aortic pannus in previously implanted mechanical AVs at the time of tricuspid valve surgery for severe TR, even if the aortic TMPG is $<40 \mathrm{mmHg}$. The initial indication for TMPR was mitral TMPG $\geq 5 \mathrm{mmHg}$. The decision to perform prophylactic TMPR regardless of a low mitral gradient was made in the operating room during AVR for patients with prominent ventricular pannus protruding into the inner side of the metal ring. TMPR was initiated from the mid-portion of the upper sewing ring after division of the pannus with a scalpel. The pannus was carefully dissected on the medial and lateral sides. Finally, the pannus was removed with a long hemostat because of the difficulty in dissecting the lower part of the MV. The procedural time was usually $1-2 \mathrm{~min}$. Occasionally, dense calcification protruding into the left ventricular cavity was removed. After pannus removal, the function of the mechanical valve was checked with a plastic valve tester. The atrial side of the mitral pannus was identified through a transseptal incision.

\section{Follow-up}

Data were collected from a computerized hospital database. Additional follow-up data were obtained through telephone interviews with the patients or family members. The national registry that maintains mortality and survival data for the Republic of Korea was searched for patients lost to hospital follow-up. Follow-up was closed on January 


\begin{tabular}{|lcc|}
\hline \multicolumn{3}{|c|}{ Table 3. $\begin{array}{l}\text { Name and Size of Previously Implanted Mechanical } \\
\text { Mitral Valves }\end{array}$} \\
Name & Size & $\mathbf{n}(\%)$ \\
ATS & $27 \mathrm{~mm}$ & $6(18)$ \\
& $29 \mathrm{~mm}$ & $4(12)$ \\
Carbomedics & $25 \mathrm{~mm}$ & $1(3)$ \\
& $27 \mathrm{~mm}$ & $2(6)$ \\
& $29 \mathrm{~mm}$ & $2(6)$ \\
& $31 \mathrm{~mm}$ & $1(3)$ \\
St. Jude & $27 \mathrm{~mm}$ & $2(6)$ \\
& $29 \mathrm{~mm}$ & $3(9)$ \\
On-X & $25 \mathrm{~mm}$ & $1(3)$ \\
Sorin & $27 \mathrm{~mm}$ & $1(3)$ \\
Duromedics & $27 \mathrm{~mm}$ & $1(3)$ \\
Medtronic-Hall & Not available & $3(9)$ \\
Not available & & $7(21)$ \\
Total & & 34 \\
\hline
\end{tabular}

31, 2017. Among the 34 patients, $26(77 \%)$ were monitored by us and $5(15 \%)$ were monitored at other hospitals. The survival data for the remaining 3 patients were obtained from the national registry. The survival follow-up was $100 \%$ complete, with a median follow-up of 89.3 months (IQR, 56.5-106.6 months). Standard guidelines were used to define morbidity and mortality. ${ }^{8}$ An early event was defined as any event occurring within 30 days of the surgery. 2D Doppler echocardiography was performed before discharge and at $1,3,5$, and $>7$ years after the surgery.

\section{Statistical Analysis}

Distribution of general characteristics, preoperative laboratory, echocardiographic data, operative data, and previously implanted mitral mechanical valve data were analyzed using mean ( \pm standard deviation; SD) or median (IQR) for continuous variables and percentage for categorical variables. Kaplan-Meier method was used to show survival curves after TMPR. To test the effects of mitral TMPG across all time points (preoperative, 1 year, and 3-5 years), we used a repeated measures ANOVA with time as the within-patient factor and group (TMPG $\geq 5 \mathrm{mmHg}$ vs. $<5 \mathrm{mmHg}$ ) as the between-patient factor.

\section{Results}

\section{Patients' and Operative Data}

The main indications of reoperative surgery were severe TR in 11 patients, severe aortic prosthetic stenosis because of pannus formation in 6 patients, severe TR with severe aortic prosthetic stenosis because of pannus formation in 9 patients, rheumatic progression in the native AV in 6 patients, malfunction of the mechanical MV in 1 patient, and paravalvular leakage of the MV in 1 patient (Table 2). In all patients, ventricular pannus was removed through the aortic root. The transaortic approach was performed after removal of the previous mechanical AV in 24 patients. The reason for repeat AVR was a stenotic mechanical AV because of subaortic pannus formation in all patients; 2 patients had associated aortic regurgitation (AR) caused by acute malfunction of the Medtronic-Hall valve, and 1 patient required extracorporeal membrane oxygenation for cardiac arrest in the emergency room. The explanted

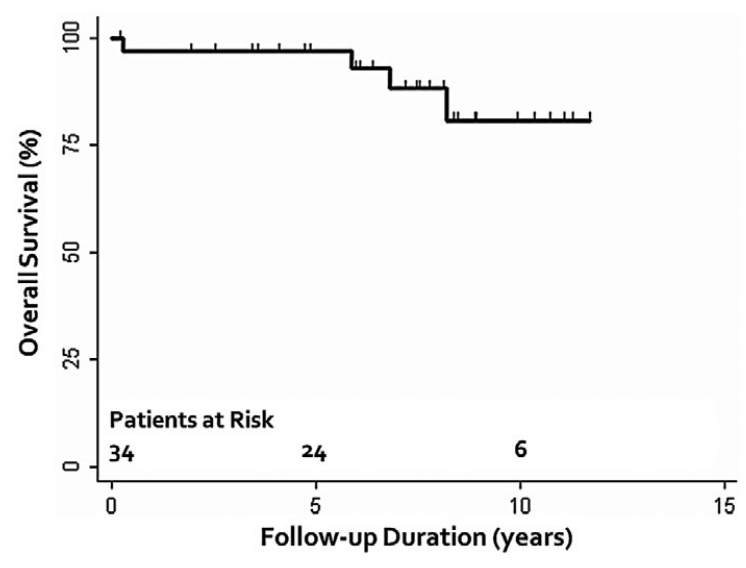

Figure 1. Kaplan-Meier survival curve after transaortic mitral pannus removal.

mechanical AVs were bileaflet valves in 14 patients and monoleaflet valves in 10 patients. In 8 patients, TMPR was performed after excision of the native diseased AV because of rheumatic progression. This approach was also used in 2 patients through a normally functioning AV. The atrial side of the mitral pannus was removed through the interatrial septum in 4 patients. The preoperative mitral TMPG was $\geq 5 \mathrm{mmHg}$ in 11 patients, including 3 patients with malfunction of the prosthetic MV. Prophylactic TMPR was performed in 23 patients with mitral TMPG $<5 \mathrm{mmHg}$; 1 patient had situs inversus totalis, mirror-image dextrocardia, and previous DVR. Repeat AVR, tricuspid annuloplasty, and prophylactic TMPR were performed by the surgeon while standing on the left side of the patient. Other concomitant operative procedures were tricuspid valve replacement (TVR) in 13, tricuspid valve repair in 15 , repeat AVR in 24, and AVR in 8 patients. The detailed operative data and names of the mechanical MVs are described in Table 2 and Table 3.

\section{Cases of Mechanical MV Malfunction}

The first case of malfunction was in a 43-year-old female patient with a history of mechanical MVR ( 9 years prior) at another hospital and thrombolytic therapy (5 years prior). In 2008, she presented with dyspnea and complete immobilization of the anterolateral leaflet of the bileaflet mechanical valve caused by thickened anterior chordae and papillary muscle. Mitral TMPG was $6.8 \mathrm{mmHg}$. Moderate to severe AR was also present. Successful transaortic removal of the thickened anterior chordae, papillary muscle, and mitral pannus after excision of the native AV was performed without repeat MVR. The second case was that of a 59-year-old female patient with a history of DVR at another hospital 17 years prior. Her preoperative mitral TMPG was $6.8 \mathrm{mmHg}$. The aortic prosthetic valve was severely stenotic because of pannus formation. Intraoperative transesophageal echocardiography showed delayed closure of 1 leaflet of the mechanical bileaflet valve. This abnormal finding disappeared after TMPR, as observed on intraoperative echocardiography. The third case was that of a 57-year-old female patient who had a history of $2 \mathrm{MV}$ surgeries. Preoperative echocardiography showed severe TR, suspected MV pannus, and mitral TMPG of $6 \mathrm{mmHg}$. 


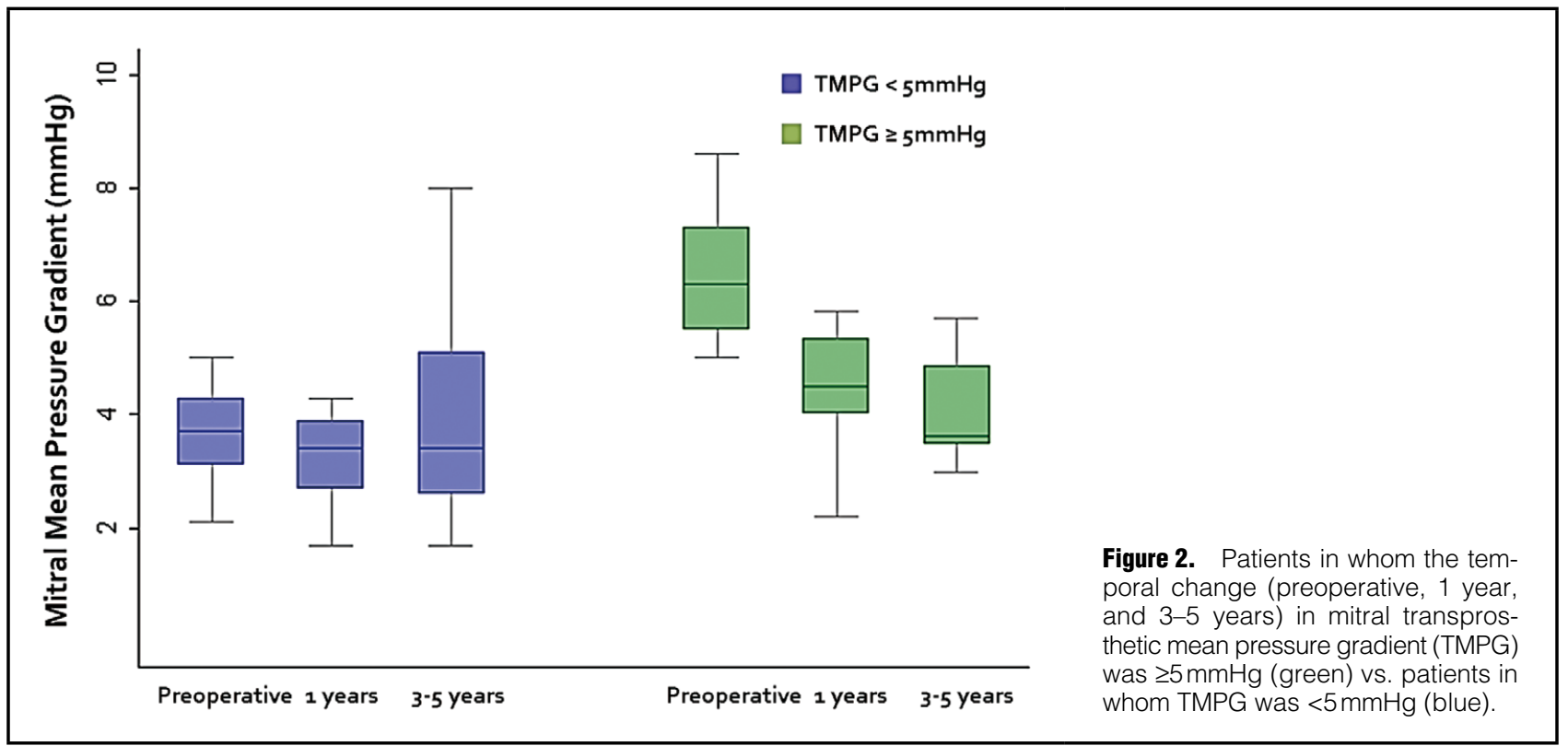

Intraoperative echocardiography showed mild eccentric MR caused by pannus overgrowth. TMPR was performed through the normally functioning AV before TVR. Intraoperative echocardiography confirmed normal function of the mechanical bileaflet valve.

\section{Early Outcome}

There were no in-hospital or 30-day deaths. Early complications included 2 permanent pacemaker implantations (1 for sick sinus syndrome, 1 for heart block), 1 reoperation because of bleeding, and 1 case of mediastinitis. The median stay in the intensive care unit and hospital was 2 days (IQR, 1.8-4 days) and 12 days (IQR, 9-16 days), respectively. All patients had normal function of the mechanical MV on echocardiography and fluoroscopy at the time of discharge. The mitral TMPG in patients with $\geq 5 \mathrm{mmHg}$ preoperatively was significantly decreased from $6.46 \pm 1.1$ to $4.37 \pm 1.17 \mathrm{mmHg}$ at the time of discharge $(\mathrm{P}<0.001)$. The mitral TMPG in patients with $<5 \mathrm{mmHg}$ preoperatively was not significantly changed $(3.61 \pm 0.86$ to $4.04 \pm 2.04 \mathrm{mmHg})$. The aortic TMPG after repeat AVR decreased from $43.9 \pm 14.6$ to $12.4 \pm 3.9 \mathrm{mmHg}$ in 24 patients $(\mathrm{P}<0.001)$.

\section{Late Outcomes}

There were 4 late deaths during follow-up: heart failure in 1 , sepsis from endocarditis in 1 , chronic renal failure in 1 , and unknown in 1 patient. The overall survival at 5 and 10 years was $97 \%$ and $81 \%$, respectively (Figure 1). Late complications included 6 cases of hemorrhage ( 5 cerebral, 1 gastrointestinal), 2 cases of endocarditis (1 reoperation, 1 medical treatment), and 2 pacemaker implantations for sick sinus syndrome. No cases of malfunction of the mechanical MV were observed on final follow-up echocardiography. New onset of AR was not detected on final follow-up echocardiography in the 2 patients with TMPR through a normally functioning AV. Mild mitral paravalvular leakage was detected in 1 patient after 8 years. Up to 5 years of serial data on mitral TMPG were available for 7 patients with $\geq 5 \mathrm{mmHg}$ and for 15 patients with $<5 \mathrm{mmHg}$ (Figure 2).

\section{Discussion}

Catastrophic malfunction of a mechanical MV because of pannus ingrowth has been extremely rare since the introduction of bileaflet valves. The reported risk factors for pannus formation in mechanical valves were small size of the valve, old type of mechanical valve (monoleaflet), young patient, female sex, rheumatic heart disease, and DVR status (aortic and mitral valves).,9-12 Pannus growth in mechanical MVs was known to occur more slowly than in mechanical AVs because of the larger size of the MV. ${ }^{9}$ Therefore, clinical symptoms associated with mitral pannus growth usually present $2-3$ decades after the initial operation.

In our previous studies, aortic TMPG was more likely to increase over time after DVR than after isolated AVR.1,2 In the aortic position, the overall TMPG increased during the 5 years after DVR. The increase in aortic TMPG was greater in female patients than in male patients. Conversely, the mitral TMPG did not change significantly during follow-up and was significantly increased only in female patients. ${ }^{3}$ Song et al reported that female sex was an independent predictor of late TR progression. ${ }^{12}$ Another report by Teshima et al showed that pannus overgrowth might be related to transforming growth factor $\beta 1$ in plasma and that the incidence of prosthetic valve dysfunction from pannus or thrombus was higher in female patients than in male patients. ${ }^{11}$ In this series, most patients were women $(88 \%)$ and had had a DVR (71\%) because of rheumatic heart pathology.

Recently, we have been replacing moderately stenotic aortic prostheses with new mechanical valves to prevent acute malfunction of the monoleaflet AV and improve right heart failure at the time of tricuspid surgery for severe TR. ${ }^{4,5}$ In our previous studies,, 3 preoperative aortic TMPG was associated with the severity of TR. Furthermore, multivariate analysis demonstrated that postoperative aortic TMPG was an independent predictor of late TR after reoperative TVR ${ }^{4}$ and $\mathrm{DVR}^{3}$. Among the 24 patients with concomitant repeat AVR in this study, 15 had severe aortic 


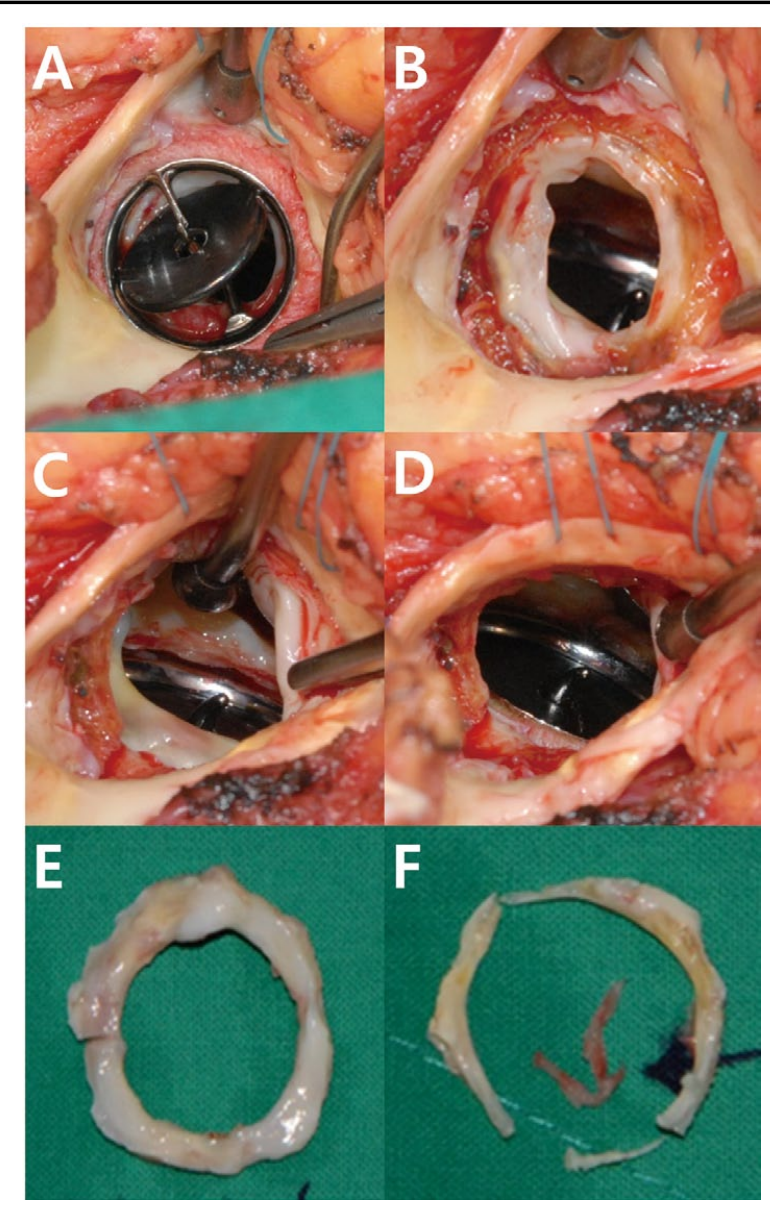

Figure 3. Transaortic mitral pannus removal in a MedtronicHall valve that was implanted 24 years prior in a 59-year-old female patient with a mitral gradient of $5 \mathrm{mmHg}$. (A,B) The aortic pannus formation is mainly located in a subaortic position below the Medtronic-Hall aortic valve. (C,D) The mechanical mitral valve is clearly seen after removal of the mitral pannus on the ventricular side. (E,F) Excised ventricular and atrial pannus. A small piece of thrombus is present on the atrial side (arrowhead)

prosthetic stenosis and 9 had moderate prosthetic stenosis. In the 9 patients with moderate stenosis and normally functioning prosthetic valve, the presence of subaortic pannus was confirmed during operation. Our indications of repeat AVR in moderate prosthetic stenosis were serial increment of aortic TMPG $>15 \mathrm{mmHg}$ during follow-up echocardiography ${ }^{1}$ or monleaflet mechanical valve regardless of preoperative TMPG. Postoperative aortic TMPG significantly decreased from $31.3 \pm 4.9 \mathrm{mmHg}$ to $12.1 \pm 3.8 \mathrm{mmHg}$ $(\mathrm{P}<0.001)$ at the time of discharge in patients with moderate aortic prosthetic stenosis. More detailed reasons for repeat AVR in patients with moderate stenosis of aortic prosthesis are described in our previous report. ${ }^{4} \mathrm{We}$ began performing TMPR in 2004 because pannus growth on the ventricular side of the prosthetic MV was frequently observed during repeat AVR in patients with previous DVR. The operative indications of TMPR were malfunction of the mechanical valve in 3 patients, mitral TMPG $>5 \mathrm{mmHg}$ in 11 patients to improve hemodynamics, and protruding mitral pannus without significant transmitral gradient at the time of redo AVR or AVR in 22 patients.

In this series, only 1 case of complete immobilization and 2 cases of minor malfunction in 1 cusp of the bileaflet mechanical MV were included. We previously reported a case of complete immobilization with successful transaortic removal of thickened anterior chordae, papillary muscle, and mitral pannus without repeat MVR. ${ }^{6}$ The 8-year follow-up echocardiogram of that patient showed no dysfunction of the preserved mechanical MV. Preservation of the entire anterior mitral leaflet during MVR should be undertaken carefully, because of early development of pannus, particularly in patients with rheumatic mitral stenosis. The other 2 cases of minor mechanical valve malfunction had abnormal intraoperative echocardiographic findings of delayed closure or eccentric mechanical MR. The mitral TMPG was $\geq 6 \mathrm{mmHg}$ in both patients. One patient required TMPR through the normal AV at the time of TVR for severe TR. Transesophageal echocardiography should be considered to detect subtle changes in the mechanical valve in patients with high mitral TMPG at the time of TV surgery.

The 1-year follow-up echocardiographic data showed that the mitral TMPG was significantly decreased in patients who had mitral TMPG $\geq 5 \mathrm{mmHg}$ preoperatively. Recently, Kim et al reported on 4 female patients who underwent transaortic pannus removal with hemodynamic improvement in a previously implanted mechanical MV.13 Their surgical technique involved transaortic removal of subvalvular pannus after pushing from the left atrium. The follow-up duration was only 3-19 months.

Based on the initial hemodynamic improvement, we extended the indications for TMPR to patients with mitral TMPG $<5 \mathrm{mmHg}$ and significant protrusion of ventricular pannus into the inlet orifice of the MV at the time of repeat AVR or AVR for a diseased native valve. Catastrophic malfunction in monoleaflet mechanical valves may be prevented or delayed with this procedure (Figure 3). In this study, we did not perform repeat MVR in 3 patients with Medtronic-Hall mitral valve, because they had good hemodynamic function and the low thrombogenicity of the Medtronic-Hall valve. Another reason to not repeat MVR with a new bileaflet valve in these patients was the increasing surgical risk in concomitant aortic and tricuspid valve surgeries. In our experience, repeat MVR in patients with pannus growth frequently requires mitral annular reconstruction with bovine pericardium after excision of the prosthetic MV. However, the Omniscience mechanical MV with pannus overgrowth was replaced with a new bileaflet mechanical valve in 2 patients because of a high risk of thrombogenicity. We performed 68 repeat MVR procedures in patients with a mechanical MV in the same time period; 18 patients had repeat MVR for pannus formation with or without thrombosis. There was 1 early death of a patient who had emergency operation for acute malfunction of a mechanical valve caused by pannus and thrombosis. All 7 patients without available data on valve name and size of mechanical MV had bileaflet valves (Table 3).

In this series, TMPR was performed through a normal functioning $\mathrm{AV}$ in 2 patients. Late follow-up echocardiography after 5 and 6 years showed no AR in either patient. Therefore, TMPR may be another option for old and sick patients with malfunction of a mechanical MV to avoid risky repeat MVR. Furthermore, elderly patients with a history of multiple reoperations frequently refuse another operation because of the high surgical risk. The recent 
trend in prophylactic tricuspid annuloplasty to prevent reoperation will be the most important issue in left-sided valve surgery for patients with rheumatic and degenerative diseases. ${ }^{14-17}$

Some studies have reported that mitral prosthesispatient mismatch (PPM) is independently associated with increased mortality, ${ }^{18,19}$ but another study found no significant effect on survival. ${ }^{20}$ Angeloni et al showed that mitral PPM is associated with the persistence of functional TR and pulmonary hypertension after MVR..$^{21}$ They recommend TV annuloplasty when PPM is anticipated at the time of MVR. In their study, the average mitral TMPG of the PPM group was $4.7 \pm 1.1 \mathrm{mmHg}$. Sato et a ${ }^{22}$ reported that PPM after MVR was a risk for overall mortality and a predictor of admission for heart failure. In their study, mean transmitral pressure gradient $\geq 5 \mathrm{mmHg}$ at 1 week postoperatively was defined as high and patients with a mean transmitral gradient $>5 \mathrm{mmHg}$ at 1 week also had significantly high mean transmitral gradient at 7 years. ${ }^{22} \mathrm{In}$ our unpublished data on serial postoperative echocardiographic follow-up in 421 patients with mechanical MVR, 64 patients had mitral TMPG $\geq 5 \mathrm{mmHg}$ at discharge. The mean TMPG of $5.8 \pm 0.9 \mathrm{mmHg}$ at discharge decreased to $4.3 \pm 1.3 \mathrm{mmHg}$ at 1 year and increased to $5.5 \pm 2.3 \mathrm{mmHg}$ at 7-year follow-up. Our results were similar to those obtained by Sato et al. We believe that a serial increase in mitral TMPG in mechanical valves is associated with pannus ingrowth in a relatively small prosthesis. Recently, cardiac computerized tomography (CT) has emerged to evaluate pannus ingrowth in mechanical aortic and mitral valves. ${ }^{23-26}$ Pannus ingrowth on CT also correlates with transprosthetic gradients on echocardiography.

This transaortic approach is also useful for removal of redundant chordae in patients with entrapment of mitral chordae after mechanical MVR with an On-X valve during which complete removal of redundant chordae is difficult through the left atrial approach because of the high profile of the mechanical valve.

The good early and late clinical outcomes in our series may be attributed to the relatively young age of the patients, fewer cases of coronary disease, good left ventricular function, early referral or decision to perform TV surgery, mostly single-surgeon experience (94\%), and intensive postoperative follow-up by the cardiac surgeon.

The clinical implications of TMPR are as follows. First, transaortic removal of previous preserved thickened mitral anterior chordae and papillary muscle head can be performed in patients with mechanical valve malfunction without a repeat MVR. Second, this transaortic approach through a normal functioning AV may be an alternative procedure for older patients in whom a repeat MVR for mechanical valve malfunction because of pannus formation carries a high surgical risk. Third, prophylactic pannus removal on the ventricular side of the mechanical MV is a feasible and safe procedure to prevent the possible fatal events of the monoleaflet mechanical MV. The prophylactic TMPR in the bileaflet mechanical MV may delay future stenosis of the valve in relatively young female patients with DVR who have a high probability of progressive pannus formation and good long-term survival. Further randomized and long-term follow-up studies are required to evaluate the clinical effects of prophylactic TMPR in the bileaflet mechanical valve.

\section{Study Limitations}

First, a small number of patients were enrolled. Second, late follow-up hemodynamic data were not available for all patients. Other hemodynamic data on measured effective orifice area of the MV were not included. Finally, we could not demonstrate the long-term benefit of prophylactic TMPR because of the short follow-up period.

In conclusion, TMPR is a safe and effective procedure for patients with malfunction of a mechanical MV or a high mitral gradient. TMPR may be considered as an alternative approach in patients with pannus overgrowth in a mechanical MV.

\section{Disclosures}

The authors have nothing to disclose with regard to commercial support. No potential conflicts exist, and no funding was provided.

\section{References}

1. Cho YH, Jeong DS, Park PW, Park KH, Sung K, Kim WS, et al. Serial changes of hemodynamic performance with Medtronic Hall valve in aortic position. Ann Thorac Surg 2011; 91: 424-431.

2. Jeong DS, Park PW, Sung K, Park SW, Lee SC, Kim WS, et al. Long-term hemodynamic performance of bileaflet prostheses versus tilting-disc prostheses in the aortic position. Int $J$ Cardiol 2013; 166: 681-687.

3. Jeong DS, Park PW, Sung K, Kim WS, Lee YT. Determinants of late tricuspid regurgitation after aortic-mitral double valve replacement. Am J Cardiol 2017; 119: 1643-1649.

4. Jeong DS, Park PW, Mwambu TP, Sung K, Kim WS, Lee YT, et al. Tricuspid reoperation after left-sided rheumatic valve operations. Ann Thorac Surg 2013; 95: 2007-2013.

5. Chang HW, Jeong DS, Cho YH, Sung K, Kim WS, Lee YT, et al. Tricuspid valve replacement vs. repair in severe tricuspid regurgitation. Circ J 2017; 81: 330-338.

6. Park B, Park PW, Park CK. Transaortic chordae and pannus removal without redo mitral valve replacement in prosthetic mitral valve malfunction. Eur J Cardiothorac Surg 2011; 39: 1057-1058.

7. Nishimura RA, Otto CM, Bonow RO, Carabello BA, Erwin JP, Guyton RA, et al. 2014 AHA/ACC guideline for the management of patients with valvular heart disease: Executive summary: A report of the American College of Cardiology/American Heart Association Task Force on Practice Guidelines. Circulation 2014; 129: $2440-2492$.

8. Akins CW, Miller DC, Turina MI, Kouchoukos NT, Blackstone $\mathrm{EH}$, Grunkemeier GL, et al. Guidelines for reporting mortality and morbidity after cardiac valve interventions. $J$ Thorac Cardiovasc Surg 2008; 135: 732-738.

9. Rizzoli G, Guglielmi C, Toscano G, Pistorio V, Vendramin I, Bottio T, et al. Reoperations for acute prosthetic thrombosis and pannus: An assessment of rates, relationship and risk. Eur J Cardiothorac Surg 1999; 16: 74-80.

10. Ellensen VS, Andersen KS, Vitale N, Davidsen ES, Segadal L, Haaverstad R. Acute obstruction by Pannus in patients with aortic medtronic-hall valves: 30 years of experience. Ann Thorac Surg 2013; 96: 2123-2128.

11. Teshima H, Fukunaga S, Takaseya T, Tomoeda H, Akashi H, Aoyagi S. Obstruction of St. Jude medical valves in the aortic position: Plasma transforming growth factor type beta 1 in patients with pannus overgrowth. Artif Organs 2010; 34: 210-215.

12. Song H, Kim MJ, Chung CH, Choo SJ, Song MG, Song JM, et al. Factors associated with development of late significant tricuspid regurgitation after successful left-sided valve surgery. Heart 2009; 95: 931-936.

13. Kim JH, Kim TY, Choi JB, Kuh JH. Haemodynamic improvement of older, previously replaced mechanical mitral valves by removal of the subvalvular pannus in redo cardiac surgery. Interact Cardiovasc Thorac Surg 2017; 24: 148-149.

14. Dreyfus GD, Corbi PJ, Chan KM, Bahrami T. Secondary tricuspid regurgitation or dilatation: Which should be the criteria for surgical repair? Ann Thorac Surg 2005; 79: 127-132.

15. Benedetto U, Melina G, Angeloni E, Refice S, Roscitano A, Comito C, et al. Prophylactic tricuspid annuloplasty in patients with dilated tricuspid annulus undergoing mitral valve surgery. J Thorac Cardiovasc Surg 2012; 143: 632-638.

16. Chikwe J, Itagaki S, Anyanwu A, Adams DH. Impact of concomitant tricuspid annuloplasty on tricuspid regurgitation, right 
ventricular function, and pulmonary artery hypertension after repair of mitral valve prolapse. J Am Coll Cardiol 2015; 65: 1931 1938.

17. Lee H, Sung K, Kim WS, Lee YT, Park SJ, Carriere KC, et al. Clinical and hemodynamic influences of prophylactic tricuspid annuloplasty in mechanical mitral valve replacement. $J$ Thorac Cardiovasc Surg 2016; 151: 788-795.

18. Magne J, Mathieu P, Dumesnil JG, Tanne D, Dagenais F, Doyle $\mathrm{D}$, et al. Impact of prosthesis-patient mismatch on survival after mitral valve replacement. Circulation 2007; 115: 1417-1425.

19. Lam BK, Chan V, Hendry P, Ruel M, Masters R, Bedard P, et al. The impact of patient-prosthesis mismatch on late outcomes after mitral valve replacement. J Thorac Cardiovasc Surg 2007; 133: $1464-1473$.

20. Jamieson WR, Germann E, Ye J, Chan F, Cheung A, MacNab JS, et al. Effect of prosthesis-patient mismatch on long-term survival with mitral valve replacement: Assessment to 15 years. Ann Thorac Surg 2009; 87: 1135-1141; discussion 1142.

21. Angeloni E, Melina G, Benedetto U, Roscitano A, Refice S, Quarto C, et al. Impact of prosthesis-patient mismatch on tricuspid valve regurgitation and pulmonary hypertension following mitral valve replacement. Int J Cardiol 2013; 168: 4150-4154.

22. Sato S, Fujita T, Shimahara Y, Hata H, Kobayashi J. Impact of prosthesis-patient mismatch on late recurrence of atrial fibrillation after cryomaze procedure with mitral valve replacement. Circ J 2014; 78: 1908 - 1914.

23. Lee S, Lee S, Pak E, Hong M, Kim J, Kim H, et al. Real-time 3D TEE for diagnosis of subvalvular pannus formation in mechanical aortic valves. JACC Cardiovasc Imaging 2015; 12: 1461-1464.

24. Teshima H, Aoyagi S, Ueda T, Takagi K, Shojima T, Tanaka H. Evaluation of advancing the standard valve dysfunction by multidetector-row CT. J Artif Org 2014; 17: 162-168.

25. Ueda T, Teshima H, Fukunaga S, Aoyagi S, Tanaka H. Evaluation of prosthetic valve obstruction on electrocardiographically gated multidetector-row computerized tomography. Circ J 2013; 77: 418-423.

26. Suh YJ, Chang B, Im DJ, Kim YJ, Hong YJ, Hong G, et al. Assessment of mitral annuloplasty ring by cardiac computed tomography: Correlation with echocardigraphic parameters and comparision between two different ring types. $J$ Thorac Cardiovasc Surg 2015; 150: 1082-1090. 